\title{
el puente KERISPER
}

\author{
R. BREFFEIL
}

Director general de la Société des Entreprises Limousin

Esta obra, situada en Bretaña (Francia), es una de las distintas que en esta zona se han construído con objeto de salvar los estuarios y reducir las distancias de los trazados de carreteras que siguen el borde del mar.

El puente se ha ubicado en el Morbihan, en la desembocadura del río Crach y en las proximidades de Trinité-surMer, donde el tráfico por carretera aumenta constantemente. La construcción del puente acorta 17 kilómetros la distancia de los 2.500 vehículos por hora que durante el verano circulan por esta carretera de orden nacional.

Como el antiguo puente fue destruído durante la última guerra y, además, como su estética y dimensiones de calzada no se ajustaban a las necesidades actuales, al proyectar la nueva obra por la Jefatura local, en colaboración con la Dirección General de París, se ha procurado salvar los inconvenientes de la anterior y ajustarla a las técnicas y necesidades modernas.

EI cauce salvado, de unos $80 \mathrm{~m}$ de anchura y profundidades de 12 a $13 \mathrm{~m}$, posee márgenes relativamente elevadas y con roca firme a poca profundidad de la tierra superficial. Esto ha permitido disponer de un buen asiento para cimientos.

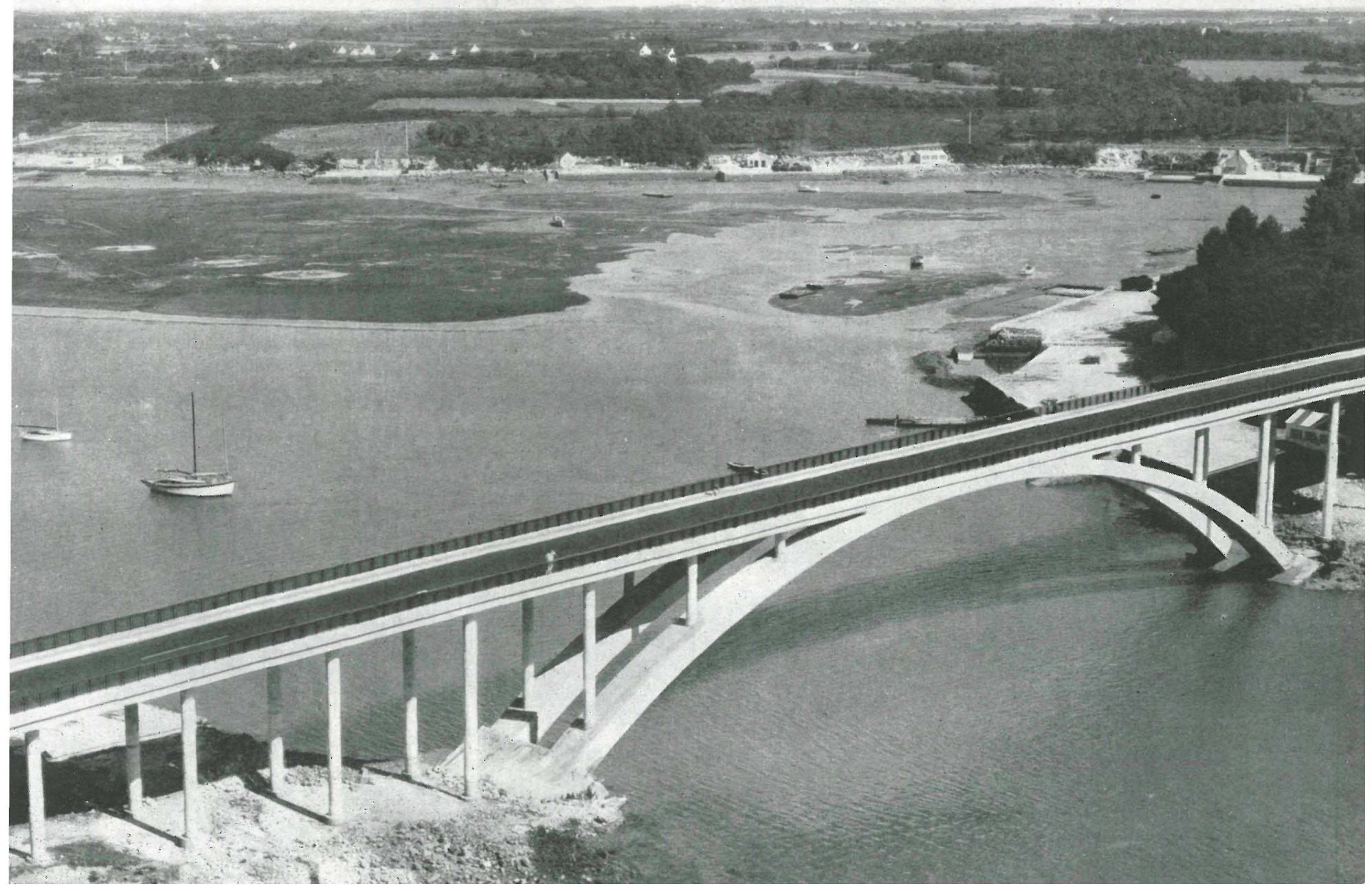



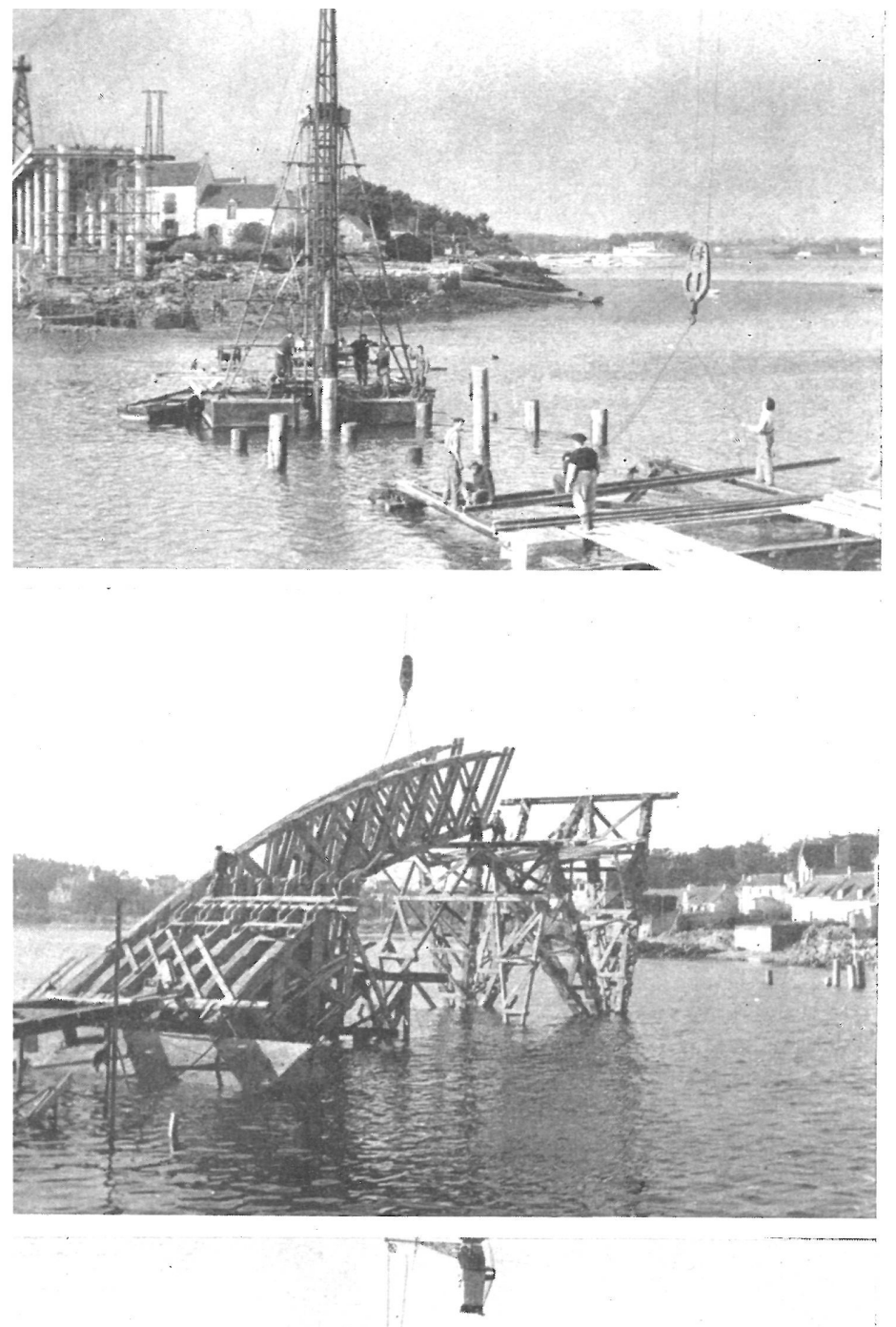

4
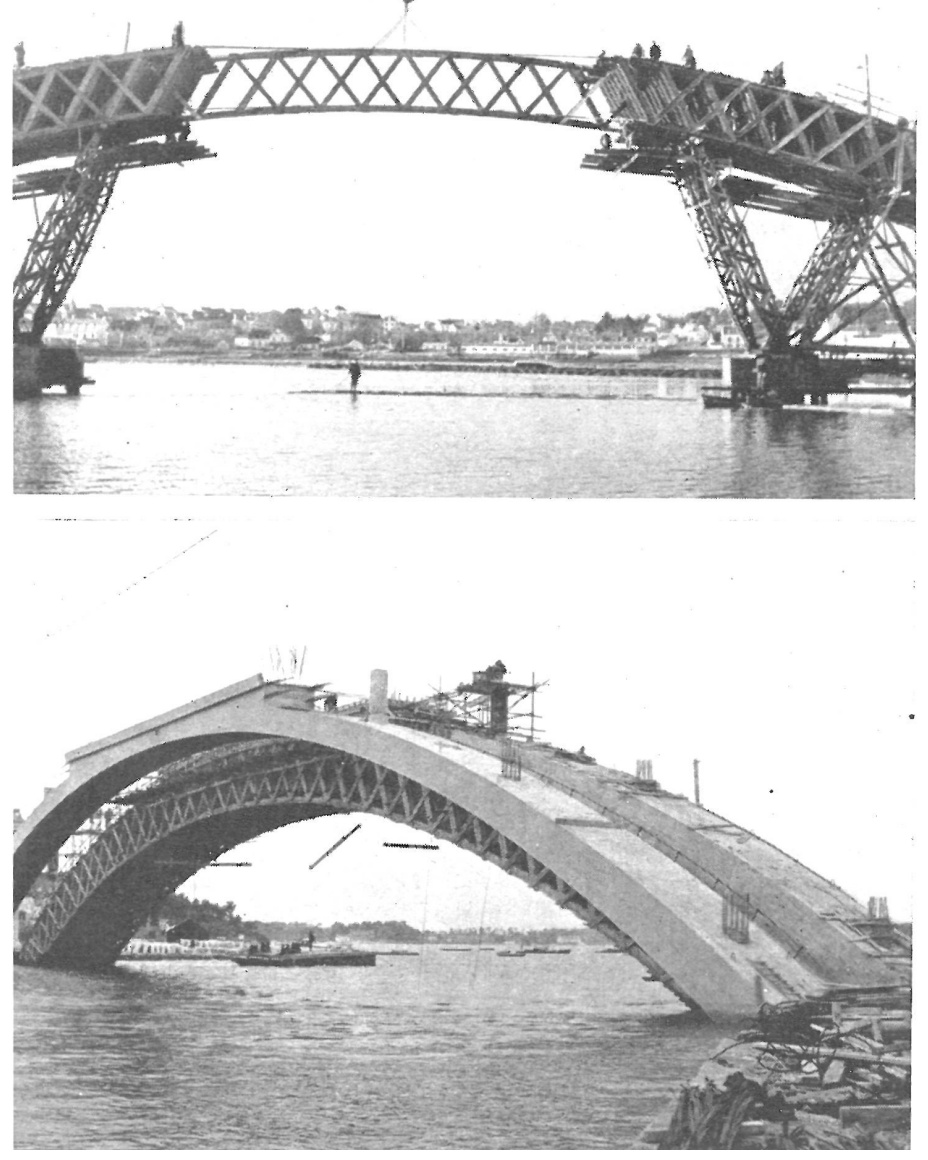

Con objeto de evitar cimentaciones en el mar, siempre engorrosas, se proyectó un arco y estribos seguidos de muros de acompañamiento para ganar en terraplén los niveles necesarios.

La obra se ha construído, en su mayor parte, de hormigón armado. El arco único tiene $86 \mathrm{~m}$ de luz y $13,75 \mathrm{~m}$ de flecha.

El tablero, de $203 \mathrm{~m}$ de longitud, se apoya sobre soportes que arrancan del arco, ligeros, formando palizada $\mathrm{y}$, sobre el terreno, los correspondientes a las extremidades del tablero. El arco se compone de dos partes independientes, cuyo conjunto constituye el único tramo. Cada una de estas partes, maciza, tiene una anchura de $2,75 \mathrm{~m}$ y un canto variable de $1,11 \mathrm{~m}$ en la clave y $1,66 \mathrm{~m}$ en los arranques. La distancia entre ejes clave y $1,66 \mathrm{~m}$ en los arranques. La distancia entre ejes
de estas dos partes o arcos parciales es de 6,25 metros.

El intradós del arco sigue, en perfil, la línea de dos arcos de elipse, de 0,82 de excentricidad, con tangente común horizontal en la clave, a la cota $14,90 \mathrm{~m}, \mathrm{y}$ que pasa por la cota 0,65 en arranques. El extradós es un arco de elipse, de igual excentricidad, que en arranques pasa por la cota $1,67 \mathrm{~m}$ y en la clave por la de 19,59 metros.

Las dos partes o arcos se han arriostrado, a uno y otro lado de la clave, en una longitud de $27,20 \mathrm{~m}$ por medio de cuatro piezas transversales y la losa. Los estribos, de hormigón armado, se apoyan directamente sobre el firme. Su anchura total es de $10 \mathrm{~m}$ y dispuestos para recibir los dos arcos.

Cinco vigas longitudinales sirven de apoyo a la losa del tablero, de $18 \mathrm{~cm}$ de espesor. La viga central y las dos intermedias tienen $0,50 \mathrm{~m}$ de anchura y 0,25 las dos laterales. La distancia entre la central y las intermedias es de $2,05 \mathrm{~m}$ y de $2,27 \mathrm{~m}$ entre una intermedia y una lateral.

Además de las cuatro piezas transversales de arriostramiento en la parte central, donde el tablero se ha bién transversal, sobre los soportes. La parte inferior de estas piezas se halla en el mismo plano que el de las vigas longitudinales laterales. La anchura de estas piezas es variable, pero se le ha dado $4 \mathrm{~cm}$ más que el diámetro de los soportes.

La distancia entre paramentos exteriores de las vigas longitudinales extremas es de $9 \mathrm{~m}$. Sobre estas vigas se ha formado una cornisa que vuela $30 \mathrm{~cm}$ y tiene $0,60 \mathrm{~m}$ de altura, extendiéndose en forma continua en toda la obra. Así dispuesto el tablero ha permitido habilitar una calzada de $6 \mathrm{~m}$ y dos andenes de 1,50 m cada uno.

La calzada está constituída por una capa de $5 \mathrm{~cm}$ de espesor de hormigón asfáltico. El encintado es de piedra natural que sobresale $18 \mathrm{~cm}$ sobre la calzada, y tiene una anchura de $20 \mathrm{~cm}$. En el espesor de los andenes se han dejado dos conductos para las canalizaciones de agua, gas y electricidad. El antepecho es metálico, pero se ha estudiado para evitar su aspecto de jaula.

Los procedimientos de cálculo empleados han sido los preconizados por el Servicio Central de Estudios Técnicos del Departamento de Obras Públicas. Consisten en el cálculo del eje sin partir de la definición analítica de la fibra media, sino a partir de las características nula fibra media, sino a partir de las características numéricas de un cierto número de secciones. Este método permite la no participación del tablero en la resistencia medio de tablas numéricas sucesivas.

El arco se ha calculado con una carga de trabajo a $100 \mathrm{~kg} / \mathrm{cm}^{2}$ para el hormigón; y en el resto de la solera, esta carga se eleva a $80 \mathrm{~kg} / \mathrm{cm}^{2}$ bajo los efectos de las sobrecargas más fuertes. La carga de trabajo previsto para el acero es de $13 \mathrm{~kg} / \mathrm{mm}^{2}$. Para el arco, las armaduras son de acero duro con carga límite de rotura de $65 \mathrm{~kg} / \mathrm{mm}^{2}$. 


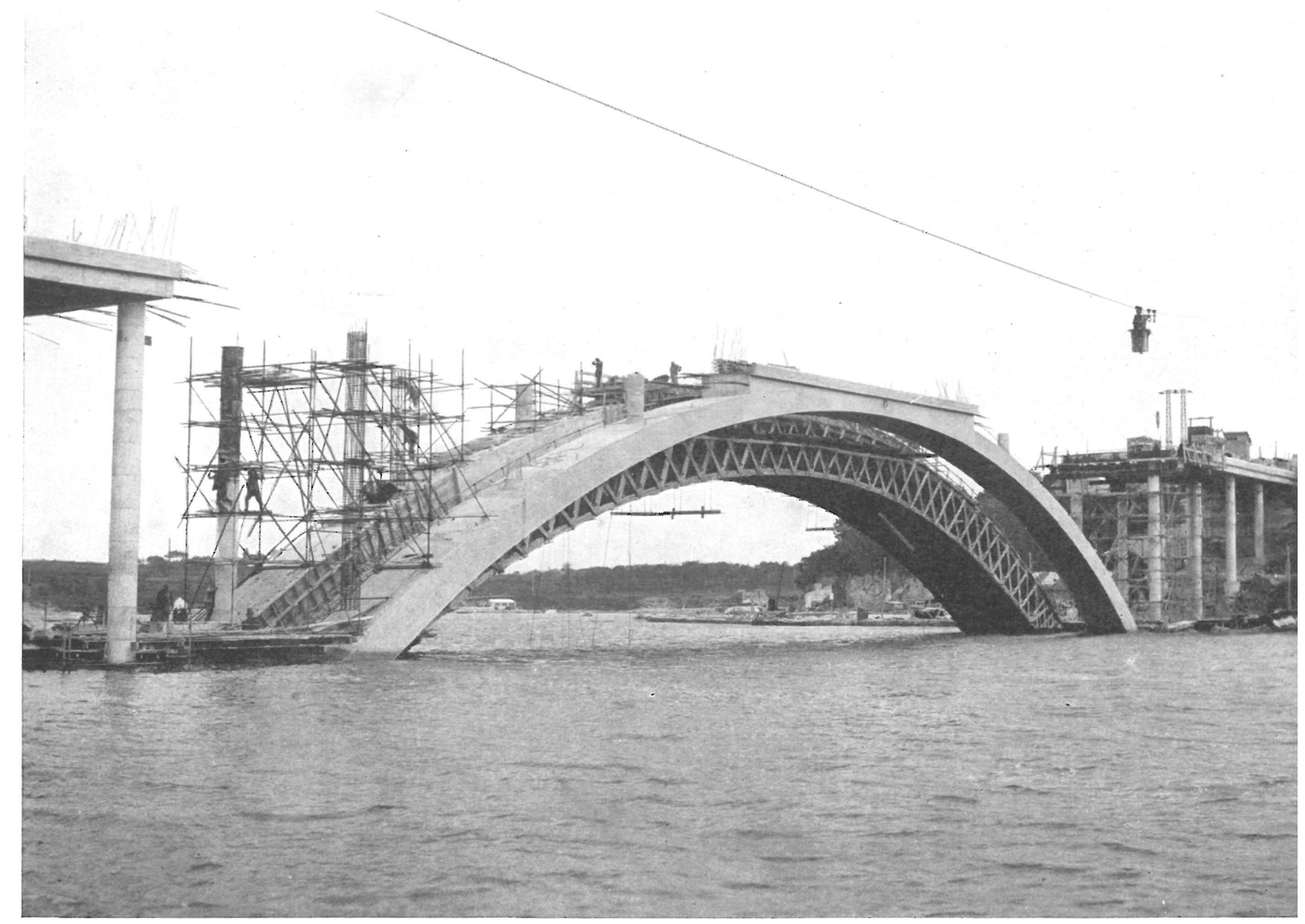

El cemento utilizado ha sido tipo portland artificial ordinario hasta la cota $+3,00$. Por debajo de esta cota, el cemento empleado es especial, es decir, cumple las condiciones impuestas para los contactos con las aguas salinas. La dosificación en cemento del hormigón ha sido de $400 \mathrm{~kg}$ para las partes por encima de la cota $+3,00$, de $450 \mathrm{~kg}$ para las partes inferiores a esta cota, y de $300 \mathrm{~kg}$ para cimientos.

Las resistencias mínimas de rotura exigidas en el Pliego de Condiciones han sido de $200 \mathrm{~kg} / \mathrm{cm}^{2}$ en el hormigón de cimientos, 300 en el tablero de $360 \mathrm{~kg} / \mathrm{cm}^{2}$ para el arco y a los noventa días. Todos estos valores han sido rebasados largamente durante el curso de la ejecución. El hormigón se ha colocado vibrando convenientemente, de preferencia en las zonas densas de armaduras, dejando una distancia de $3,5 \mathrm{~cm}$ entre éstas y los paramentos con objeto de contar con las pérdidas que el bujardeo introduce.

Han sido objeto especial de cuidado los encofrados, ya que se había previsto bujardar la cornisa, partes vistas de las vigas longitudinales y muros de sustentación, utilizando para ello bujardas accionadas con aire comprimido. Los paramentos de los soportes se han dejado como salieron de los encofrados, pero éstos, como se dijo anteriormente, se han cuidado mucho para lograr un aspecto satisfactorio.

Durante la ejecución ha habido que vencer algunas dificultades, entre las cuales se halla la necesidad de dejar un paso libre de $25 \mathrm{~m}$ de anchura, en la zona de aguas profundas, para la navegación y tráfico del puerto, los inconvenientes que arrastra el no disponer de puntos fijos de apoyo intermedio para la cimbra y la necesidad de limitar a un mínimo los entramados y andamios con objeto de oponer pequeñas resistencias a los vientos veloces que se levantan en estas regiones tormentosas.

La parte más original de la obra durante la construcción ha sido la cimbra, encargada de soportar los encofrados de los arcos. Esta cimbra se formó con un arco peraltado, de madera, encastrado en los estribos, que constituían un apoyo indeformable. Esta cimbra se preparó para el volteo de un solo arco, por lo que, terminado el primero, se ripó y se utilizó para hormigonar el segundo. El movimiento lateral de corrimiento de la cimbra fue de unos 7 metros.

Al recibir la carga del hormigón, la cimbra, tipo particular proyectado por la Empresa Limousin, está constituída por siete cerchas trianguladas, que constituyen un arco de $5,65 \mathrm{~m}$ de anchura y $3,10 \mathrm{~m}$ de altura, ancladas sólidamente en los arranques del arco, y, de tal forma, aue permiten un fácil descimbrado y corrimiento posterior para hormigonar el segundo arco. El arriostramiento entre cerchas se ha realizado con tabla de $4 \mathrm{~cm}$ de espesor. Este arriostramiento, en la parte superior, se compone de dos capas de tabla de 2,7 cm de espesor; la primera celosía y la segunda a tope. El arriostramiento en la parte inferior se compone de una sola capa de tabla dispuesta en celosía. 


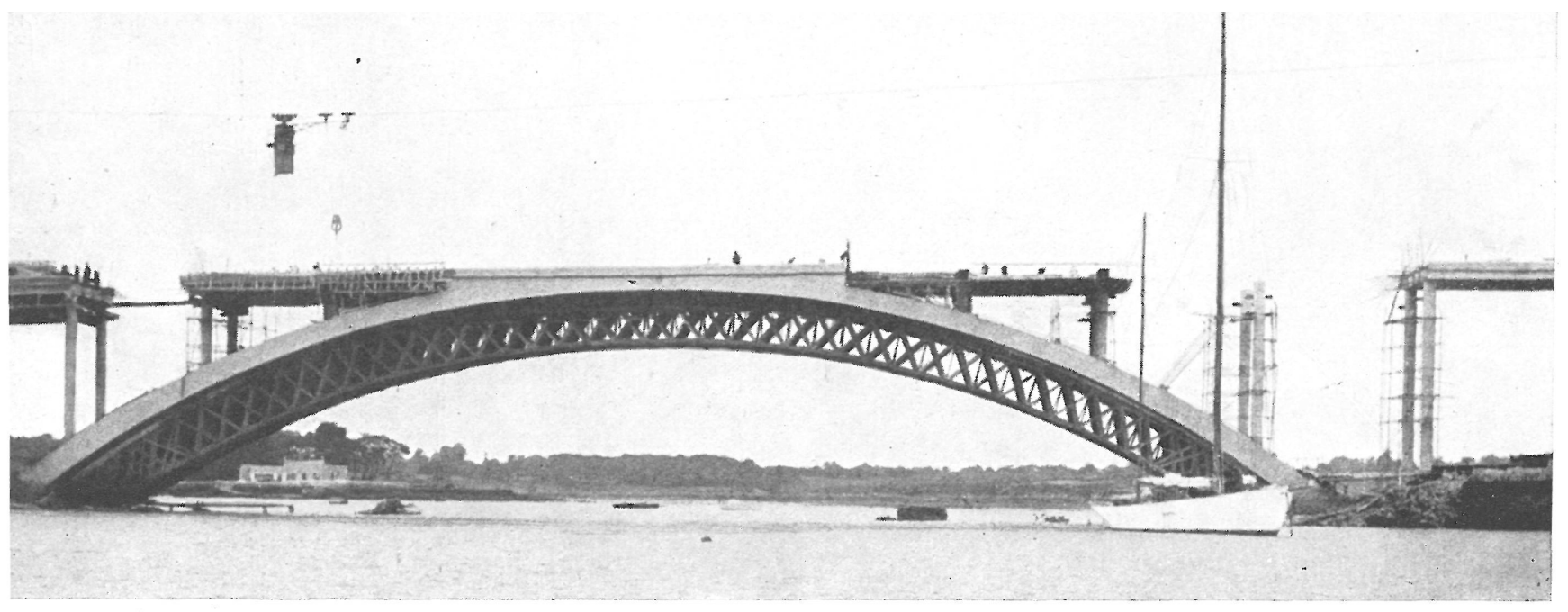

La cimbra presenta la particularidad de hallarse sometida a los efectos de la marea, lo que hacía muy difícil el empleo de cajas de arena para descimbrar. Con objeto de prever la transmisión del empuje, la estabilidad con tiempo ventoso y el ajuste necesario para el descimbrado, la cimbra transmite los empujes a los estribos por medio de calas inclinadas de hormigón armado y con gatos que sólo intervienen en el descimbrado después de colocadas las calas de hormigón. La estabilidad, a los efectos del viento, se asegura por medio de cables que solidarizan los estribos con la cimbra y unas zapatas con hormigón armado de apoyo directo de la cimbra. Estas zapatas tienen cavidades para alojar los gatos verticales que sólo sirven para soportar el peso de la cimbra y para el descenso de la misma hasta el dispositivo utilizado para su corrimiento lateral.

Después de montar la cimbra se procedió al hormigonado del arco. Como el espesor era de consideración y no se podía abusar del propio peso, se procedió por capas sucesivas; en cada una de ellas, de 0,50 m de espesor, se subdividió el arco en cuatro dovelas o trozos principales, procediendo en forma tal que se repartiese simétricamente la carga. La primer dovela fue la correspondiente a la clave; la segunda, a los riñones; la tercera, al espacio libre entre las otras dos, y la cuarta, a los arranques. Finalmente, se hormigonó la unión entre arranque y la parte en que se apoya el arco en los estribos. Para hormigonar la segunda capa se procedió en forma similar y con los mismos cuidados.

Para descimbrar se empezó destruyendo las calas de hormigón armado con objeto de cargar el empuje sobre los gatos inclinados. Después se procedió a poner en carga los gatos hidráulicos, y se fue cediendo hasta que la cimbra estuviese cargada únicamente con su propio peso. Cuando la cimbra se encontraba en estas condiciones, se colocó el tirante provisional, se aflojaron los gatos inclinados, se montaron los verticales y se descendió la cimbra sobre el dispositivo preparado para su corrimiento lateral con ayuda de dichos gatos.

El corrimiento lateral se llevó a cabo con una vía, sobre la que se colocaron rodillos de $80 \mathrm{~mm}$. Durante el corrimiento de la cimbra se utilizaron vientos anclados en el arco recién descimbrado para asegurar la estabilidad. La colocación de la cimbra en el segundo arco se realizó siguiendo los mismos procedimientos que en el primero.

En el cálculo de la cimbra se tuvo en cuenta el peso propio, la primera capa de hormigón, la variación de temperatura, las acciones del viento y deformaciones. También se calculó la influencia que podría tener sobre la cimbra el hormigonado de la segunda capa y la contraflecha que debía darse para corregir los defectos de la deformación, apreciada en unos $3 \mathrm{~cm}$ para la cimbra y $1,2 \mathrm{~cm}$ para el arco.

Para la ejecución se empezó montando el cable transportador que debía utilizarse en el transporte de materiales y hormigonado. Con ayuda de este poderoso medio auxiliar, se pudo terminar la obra después de cinco meses y medio de haber empezado el montaje de la cimbra.

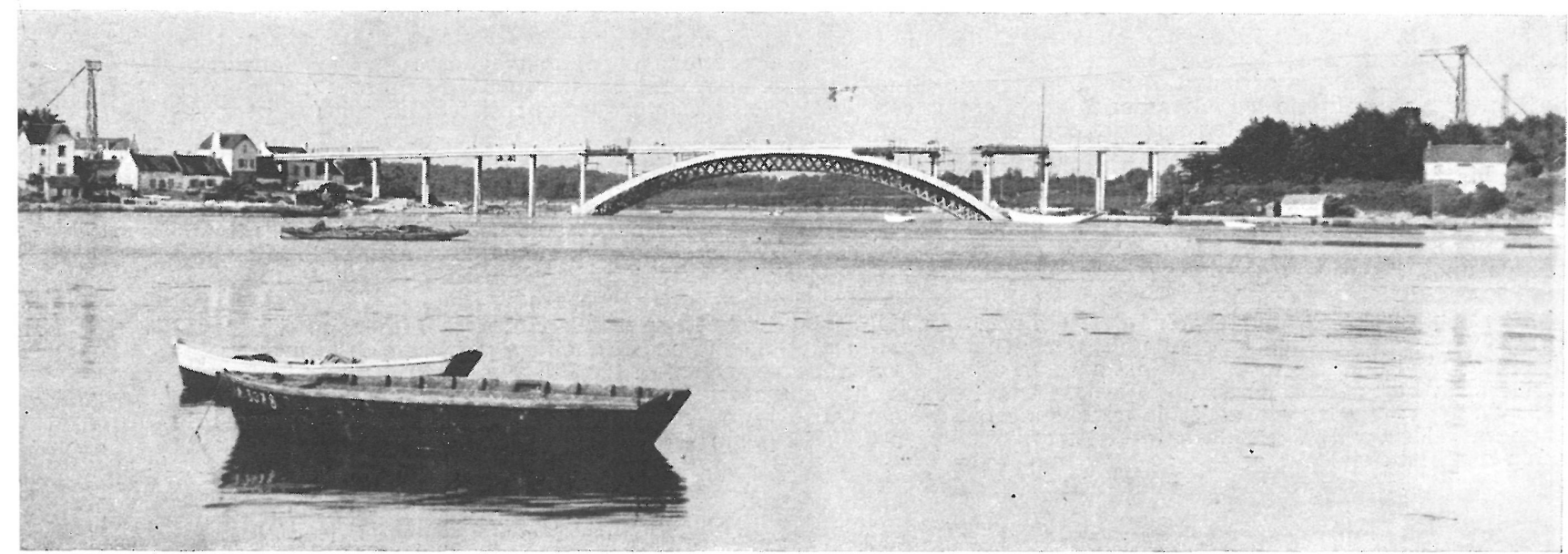

